\title{
Real-time polymerase chain reaction for detection of low-intensity Schistosoma japonicum infections in China
}

Lier, Tore; Simonsen, Gunnar S; Wang, Tianping; Lu, Dabing; Haukland, Hanne H.;

Vennervald, Birgitte J; Hegstad, Joachim; Johansen, Maria Vang

Published in:

American Journal of Tropical Medicine and Hygiene

Publication date:

2009

Document version

Publisher's PDF, also known as Version of record

Citation for published version (APA):

Lier, T., Simonsen, G. S., Wang, T., Lu, D., Haukland, H. H., Vennervald, B. J., Hegstad, J., \& Johansen, M. V. (2009). Real-time polymerase chain reaction for detection of low-intensity Schistosoma japonicum infections in China. American Journal of Tropical Medicine and Hygiene, 81(3), 428-432.

http://www.ajtmh.org/cgi/content/abstract/81/3/428 


\title{
Real-Time Polymerase Chain Reaction for Detection of Low-Intensity Schistosoma japonicum Infections in China
}

\author{
Tore Lier,* Gunnar S. Simonsen, Tianping Wang, Dabing Lu, Hanne H. Haukland, Birgitte J. Vennervald,
} Joachim Hegstad, and Maria V. Johansen

Department of Microbiology and Infection Control, University Hospital of North Norway, Troms $\phi$, Norway; Department of Microbiology and Virology, Faculty of Medicine, University of Troms $\varnothing$, Troms $\varnothing$, Norway; Anhui Institute of Parasitic Diseases, Wuhu, People's Republic of China; Department of Infectious Diseases, Faculty of Medicine, Imperial College, London, United Kingdom; DBL-Centre for Health Research and Development, Faculty of Life Sciences, University of Copenhagen, Copenhagen, Denmark

\begin{abstract}
More sensitive methods for diagnosing infection with Schistosoma japonicum are needed as control becomes more effective. We compared a real-time polymerase chain reaction (PCR) for stool samples with conventional diagnostic methods in a study of 1,727 persons from Anhui Province, China. Seroprevalence determined by using an indirect hemagglutination assay (IHA) was much higher $(26.1 \%)$ than the prevalence in stool-based tests, which were $5.3 \%, 3.2 \%$, and $3.0 \%$ for PCR, hatching test, and Kato-Katz thick smear, respectively. A large proportion of the positive stool samples were only positive in one or two tests. The PCR showed better agreement with IHA than the other two stool-based tests. A commonly used diagnostic algorithm with initial screening for antibodies and subsequent testing with the Kato-Katz thick smear of the seropositive results would have resulted in treatment of 22 people compared with 50 people if the PCR replaced the Kato-Katz thick smear. As prevalence and intensity decrease, the benefit of increased sensitivity using the PCR must be weighed against additional costs.
\end{abstract}

\section{INTRODUCTION}

Despite tremendous success in controlling Schistosoma japonicum in the past half century, the parasite is still a major public health concern in schistosomiasis-endemic countries, with China having the most cases. ${ }^{1,2}$ In most of the villages where infection occurs, the prevalence and average infection intensity is now low. ${ }^{3}$ This finding creates a diagnostic dilemma because the usefulness of diagnostic tests commonly used in China, such as the Kato-Katz thick smear, the hatching test, and antibody detection, changes with the epidemiology of the disease. When endemicity is high, morbidity control is usually attempted through mass chemotherapy. ${ }^{3-5}$ Under such circumstances, it is cost-effective to use a simple and cheap diagnostic test. High sensitivity is less important, and even high specificity is not vital because praziquantel is inexpensive and relatively safe. When the level of endemicity decreases, transmission control and interruption are desirable goals, but these strategies will accordingly require more sensitive and specific diagnostic tests. Inferior test performance will compromise the positive predictive value, which is vital for the design and execution of control efforts. ${ }^{6}$

Kato-Katz thick smears are easy, quick, quantitative, inexpensive to perform, and have for several decades been successfully used to diagnose schistosomiasis and other helminth infections in highly endemic areas. ${ }^{7}$ However, it has repeatedly been shown that the sensitivity of the Kato-Katz technique decreases with decreasing intensity of S. japonicum infection. ${ }^{8,9}$ For this reason, antibody detection in serum has been extensively used, especially the indirect hemagglutination assay (IHA) and the enzyme-linked immunosorbent assay with soluble egg antigen. ${ }^{10-12}$ The main problem with this approach has been low specificity because of persistent antibodies from previous infections and cross-reactions with other helminth infections. A commonly used diagnostic strategy has therefore been to screen for antibodies and subsequently examine the stool of

* Address correspondence to Tore Lier, Department of Microbiology and Infection Control, University Hospital of North Norway, PO Box 56, N-9038 Troms $\varnothing$, Norway. E-mail: tore.lier@unn.no seropositive persons by using the Kato-Katz or hatching test. ${ }^{3}$ Only those persons who have positive results in the latter tests are treated.

The polymerase chain reaction (PCR) has the potential for high sensitivity and specificity. Pontes and others reported the first use of PCR for detection of $S$. mansoni in a population study. ${ }^{13}$ A few Schistosoma PCR assays for diagnosing human cases have been reported, but with a single exception, they have all been applied only to a limited number of clinical samples. ${ }^{14}$ This finding is also true for three PCR assays used for detection of $S$. japonicum in humans. ${ }^{15-18}$ Thus, the epidemiologic situation makes the search for improved diagnostic tests especially relevant for this species. We evaluated a probebased real-time PCR as a diagnostic alternative and compared it with the Kato-Katz test, the hatching test, and the IHA with clinical samples from China.

\section{MATERIALS AND METHODS}

Study population and sample collection. The study population was recruited from the Guanghui and Heping villages in Laozhou Township, which is located on an island in the Yangtze River in Tongling County, Anhui, China. Serum was collected in capillary tubes from the ear lobe. The Kato-Katz test, the hatching test, and the PCR were performed with one stool sample. The Kato-Katz test, the hatching test, and the IHA were performed by experienced technicians at the Anhui Institute of Parasitic Diseases. Extraction of DNA for the PCR was conducted at the same institute, and PCR amplification was performed at the institution of the first author. Informed consent was obtained from the participants. Ethical approval was provided by the village leadership, the Scientific Committee at Anhui Institute of Parasitic Diseases, and the Tongling County Health Bureau. Participants who were positive were treated with one oral dose of praziquantel (40 mg/kg) (Nanjing Pharmaceutical Factory, Nanjing, China).

Indirect hemagglutination assay. Antibody detection was performed by using the IHA and a kit containing human erythrocytes coated with soluble egg antigen. The kit is commercially available from the Anhui Provincial Institute of 
Parasitic Diseases (Wuhu, China). The test procedure was as described by Zhou and others. ${ }^{12}$ The test result was considered positive when a positive reaction appeared at a titer $\geq 1: 10$.

Real-time PCR. Plastic spoons were used to measure a stool volume equivalent to one gram. DNA was extracted by the use of rapid one-step extraction buffer and ethanol precipitation as described. ${ }^{17,18}$ The PCR was based on a previously evaluated assay targeting the mitochondrial NADH dehydrogenase I gene, but with new primers and probe-based detection to maintain high specificity. ${ }^{17,18}$ The consensus sequence from alignment of NADH dehydrogenase I gene sequences from S. japonicum (GenBank accession nos. AF215860, AF05629196, and AF056288-90) covering seven geographic locations in China and The Philippines were used as basis for primer and probe design. ${ }^{19-21}$ Alignment was performed using SeqMan version II 5.05 Lasergene software (DNASTAR, Madison, WI).

The new primers and probe were designed using Primer Express version 3.0 software (Applied Biosystems, Foster City, CA). The primer set F-SJ-ND1 (forward: 5'-ACTGGTTA TGGTTTGTTGATGTTAGGT-3') and R-SJ-ND1 (reverse: 5'-AGCCACACGAACAGCACTAATC-3') synthesize a 75-basepair amplicon. The probe was a minor groove binding probe, MGB-SJ-ND1 (Applied Biosystems): 6-FAM-5'AGGTTCTTGGAAAAAGTAT-3'-MGBNFQ (MGBNFQ is a minor groove binder/non-fluorescent quencher). The reaction mixture consisted of $5 \mu \mathrm{L}$ of DNA template, $12.5 \mu \mathrm{L}$ of TaqMan Universal PCR Master Mixture (Applied Biosystems), $300 \mathrm{nM}$ of each primer, $250 \mathrm{nM}$ of probe, $2 \mu \mathrm{L}$ of bovine serum albumin (Promega, Madison, WI), and distilled water to give a final volume of $25 \mu \mathrm{L}$.

The cycle protocol consisted of incubation at $50^{\circ} \mathrm{C}$ for 2 minutes, $95^{\circ} \mathrm{C}$ for 10 minutes, and 50 cycles at $95^{\circ} \mathrm{C}$ for 15 seconds and $60^{\circ} \mathrm{C}$ for 1 minute. A 7300 Real Time PCR System thermal cycler with accompanying SDS software (both from Applied Biosystems) was used. The PCRs were carried out in duplicate for every sample. Samples that were positive in only one PCR were tested in triplicate, and the test result was regarded as positive when at least one of these triplicate results was positive. As a result of this modification, 12 samples had to be retested, of which 5 were regarded as having positive results after the second run. We found the PCR amplification curves easy to classify into positive and negative results. Non-template controls, with water instead of DNA template, were included in all runs to check for contamination.

Sensitivity for different egg counts was examined by spiking one-gram stool samples with various numbers of S. japonicum eggs recovered from mice livers. ${ }^{17}$ The 46 samples contained 1,000 eggs $(n=2), 100$ eggs $(n=8), 50$ eggs $(n=13), 10$ eggs $(n=7), 5$ eggs $(n=7)$, and 1 egg $(n=9)$. The PCR result was positive for all the samples except for one sample that contained one egg per gram (epg) of stool.

The PCR specificity was tested in three types of material. The first type was DNA extracted from adult worms of the following species: S. japonicum (Anhui strain), S. mansoni, S. haematobium, S. bovis, and Opisthorchis viverrini. The $S$. japonicum strain was PCR positive, and the other Schistosoma species and $O$. viverrini were PCR negative. The second type was the study database that was searched for samples that were Kato-Katz positive for other helminth eggs, but negative for S. japonicum by the Kato-Katz test, the hatching test, and the IHA. We found 6 samples containing Ascaris lumbricoides and 34 containing Trichuris trichiura. All 40 samples were PCR negative. The third type was DNA extracts from 29 bacterial and yeast species in stool (all from American Type Culture strains; LGC Standards AB, Boras, Sweden) and human DNA from two cell lines (MRC-5 and HE). All 31 samples were PCR negative.

Hatching test and Kato-Katz thick smear. The hatching test was conducted by transferring approximately 30 grams of stool into a metal container with a coarse metal mesh. Fine material was flushed into a fine-meshed nylon bag by using flowing water and a stirring stick. Sediment was washed by flushing with water until the water was clear and by stroking the outside of the bag with chopsticks. The content of the bag was then transferred to an Erlenmeyer flask containing $300 \mathrm{~mL}$ of non-chlorinated water from the Yangtze River. The flasks were left in a well-lit room at approximately $28^{\circ} \mathrm{C}$ and checked by two of the staff for swimming miracidia after $4,6,8$, and 24 hours by using a strong light that illuminated the back or side of the flasks.

The Kato-Katz thick smear was performed as described by using nylon screens and plastic templates (Zhejiang Ningbo Medical Instrument Factory, Ningbo, China). ${ }^{11}$ Three slides (41.7 $\mathrm{mg}$ each) were prepared from one stool sample from each person and examined within a week.

Data analysis. Data were stored in an Excel 2002 spreadsheet (Microsoft Corp., Redmond, WA) and analyzed with SPSS version 14.0 for Windows software (SPSS, Inc., Chicago, IL). Only stool samples with results from all three stool-based tests were selected to examine the agreement between the three tests $(\mathrm{n}=1,106$; Figure 1$)$. The value of $\kappa$ was calculated using $(\mathrm{Po}-\mathrm{Pe}) /(1-\mathrm{Pe}) .^{22}$

To calculate effects on patient treatment by changing from a traditional IHA/Kato-Katz-based algorithm to a strategy based on the IHA and PCR, we selected all samples with available results for IHA, Kato-Katz, and PCR $(\mathrm{n}=942)$.

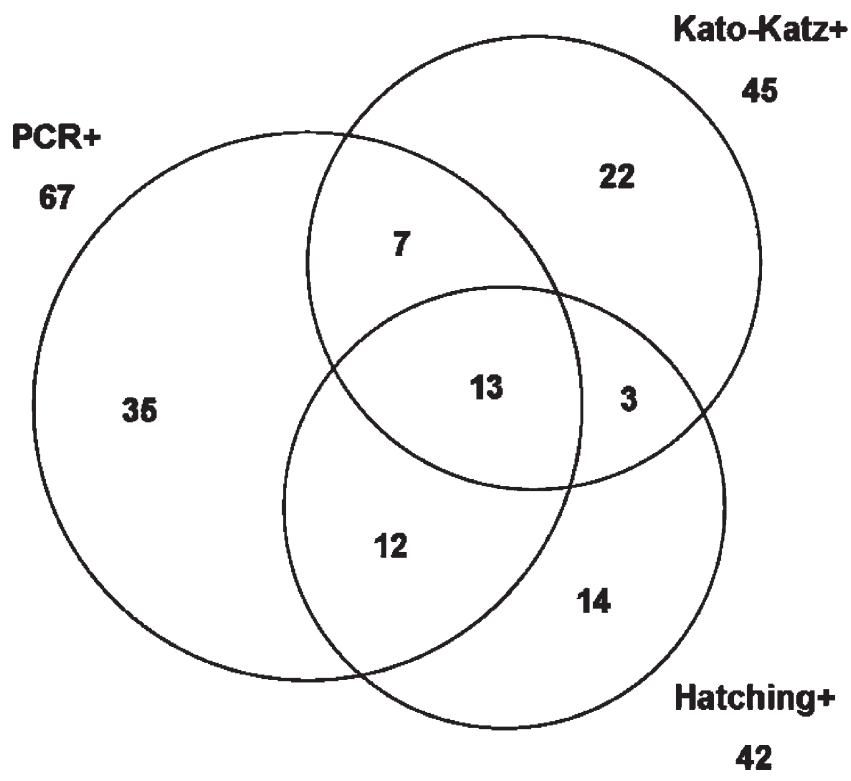

Figure 1. Agreement between polymerase chain reaction, KatoKatz thick smear, and hatching test for stool-based diagnosis of Schistosoma japonicum infections, China. Values indicate the number of positive samples in each group. 


\section{RESULTS}

Prevalence and egg counts. Samples were collected from 1,727 persons $(52 \%$ males and $48 \%$ females, age range $=6-84$ years, mean $=40$ years). Results from all diagnostic tests were not available for the whole study population. The total number of samples tested, number of positive samples, and prevalence of $S$. japonicum determined by using the three diagnostic tests are shown in Table 1.

The 46 Kato-Katz-positive samples could be divided into 3 egg count groups: 26 samples $(57 \%)$ with $<40$ epg, 15 samples $(33 \%)$ with 40-99 epg and 5 samples $(11 \%)$ with $>99$ epg. The lowest possible egg count in this study was 8 epg because this value represents 1 egg per 3 Kato-Katz slides. There were 9 samples with 8 epg and 7 with 16 epg (total of 2 eggs per 3 slides).

Agreement between tests. The degree of agreement between positive results from the three stool-based tests is shown in Figure 1. A total of $52 \%$ of the PCR positive samples were positive only by the PCR. The corresponding values for the Kato-Katz and hatching tests were $49 \%$ and $33 \%$, respectively. Calculation of the strength of agreement resulted in a value of $\kappa=0.32$ the between the PCR and Kato-Katz test, 0.43 between the PCR and hatching test, and 0.34 between the Kato-Katz and hatching tests.

Agreement between IHA and stool-based test results is shown in Figure 2. This figure shows the proportion of positive IHA results in samples positive in each of the three tests and the proportion of positive IHA results in samples where one test result was positive and the other test result was negative.

The proportion of positive IHA results increased with increasing egg count. In samples with less than 20 epg $(\mathrm{n}=13)$ $31 \%$ were IHA positive, with $20-100$ epg $(n=24) 58 \%$ were IHA positive, and with > $100 \mathrm{epg}(\mathrm{n}=5) 80 \%$ were IHA positive.

Treatment algorithm. If the Kato-test had been used in a traditional treatment algorithm (screening with IHA and testing the seropositive samples with the Kato-Katz test), it would have resulted in treatment of 22 persons. Nineteen patients who were Kato-Katz positive but IHA negative would not have received treatment. If the PCR had been used as the stool-based test, 50 persons would have been treated, and 13 persons who were PCR positive but IHA negative would not have been treated.

\section{DISCUSSION}

Consistent with the increasing success of the schistosomiasis control program in China, making a reliable diagnosis with the methods used has become increasingly problematic. In search of a diagnostic alternative, we compared a real-time PCR with the Kato-Katz thick smear, hatching test, and IHA, which are

TABLE 1

Prevalence of Schistosoma japonicum infections using different serologic and stool-based diagnostic tests, China*

\begin{tabular}{lcc}
\hline \multicolumn{1}{c}{ Test } & No. of samples tested & No. positive (\%) \\
\hline PCR & 1,295 & $68(5.3)$ \\
Kato-Katz & 1,531 & $46(3.0)$ \\
Hatching & 1,535 & $49(3.2)$ \\
IHA & 1,195 & $312(26.1)$ \\
\hline
\end{tabular}

* PCR = polymerase chain reaction; IHA = indirect hemagglutination assay.

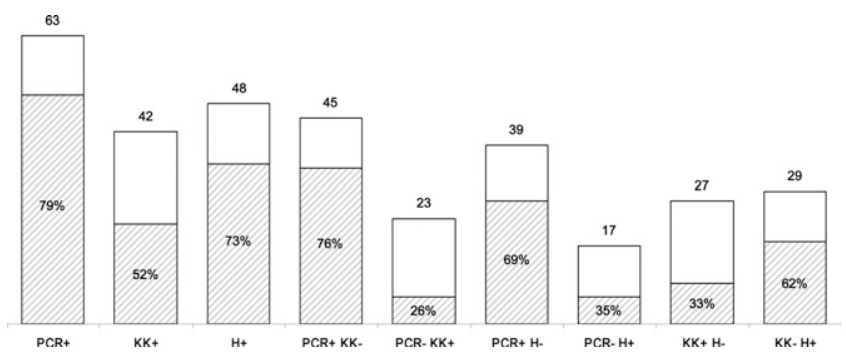

FIgURE 2. Agreement between the indirect hemagglutination assay (IHA) and stool-based polymerase chain reaction, Kato-Katz thick smear (KK), and hatching test $(\mathrm{H})$ for Schistosoma japonicum egg detection, China. The size of each column corresponds to the number of positive stool-based samples in each group. The percentage in each column represents the proportion positive by IHA in the group.

diagnostic tests commonly used in the schistosomiasis control program in China. In a large nationwide survey in China during 2004, the average $S$. japonicum prevalence in humans was $2.5 \%$ in villages classified as endemic for schistosomiasis. In more than half of the villages, the prevalence was $<1 \%$. The average egg output was 33 epg. ${ }^{3}$ Such low prevalence and low egg output is a huge challenge for use of diagnostic tests. Performance of diagnostic tests is often evaluated in populations with higher S. japonicum prevalence than what is now most common in China., ${ }^{9,23-25}$ The predictive value of a test cannot automatically be transferred between two populations with different prevalences.

In the present study, the prevalence ranged from $3.0 \%$ to $5.3 \%$, depending on the stool-based test used. The PCR test gave a higher prevalence of S. japonicum infections than the Kato-Katz and hatching tests. Pontes and others reported the prevalence of $S$. mansoni to be $30.9 \%$ in 3 stool samples examined with duplicate Kato-Katz smears in contrast to $38.1 \%$ in one sample examined by PCR. ${ }^{13}$ In Senegal, ten Hove and others found that a PCR with two stool samples resulted in lower $S$. mansoni prevalence ( $73 \%$ versus $80 \%$ ) than the Kato-Katz test with the same two samples (duplicate 25-mg smears from each sample). ${ }^{14}$ However, these results can not be compared directly to the present study because the assay and the epidemiology are different.

A surprising finding in our study was the low overall agreement between the three stool-based tests and their large variation in concordance with the IHA. The total number of positive samples detected by the Kato-Katz and hatching tests was almost the same, but the results corresponded in less than half of all cases. Low agreement between S. japonicum diagnostic tests was also evident in earlier studies. ${ }^{11,12}$

Several explanations may account for the discrepancies in the results among the different test methods. The reproducibility of test results will be compromised when egg content in each sample is close to the detection limit of the tests. In the present study, $35 \%$ of the Kato-Katz-positive samples had only one or two eggs on 3 slides $(<20$ epg). The PCR uses a larger sample than the Kato-Katz test, but even one gram amounts to only approximately $0.6 \%$ of the daily stool output in a person in China. ${ }^{26}$ An even larger amount of stool is examined in the hatching test, but hatchability, and thus sensitivity of the assay, may be influenced by a number of ill-defined factors. ${ }^{11,27}$ Finally, the uneven distribution of S. japonicum eggs within stool samples may further lead to discrepancies between test results. ${ }^{8,28}$ 
An alternative explanation for discordant test results may be low specificity for one or more of the tests, leading to falsepositive results. Figure 2 shows that IHA results correlate better with PCR results than with Kato-Katz results. The Kato-Katz test is often used as a diagnostic gold standard and is regarded as having close to absolute specificity. ${ }^{11,12}$ Brinkmann and others have shown that this finding is not always the case in large-scale control programs for S. mansoni and S. haematobium..$^{29}$ Because $S$. japonicum egg are round and have fewer conspicuous characteristics than $S$. mansoni eggs, we consider it plausible that misdiagnosis may occur, especially in cases with only 1 egg on 3 slides. In the present study, we found better agreement between the Kato-Katz test and IHA in samples with high egg counts than in samples with low egg counts. This finding may indicate a higher number of false-positive Kato-Katz test results in the group with low egg counts. An alternative explanation is that IHA has lower sensitivity when egg counts are low. ${ }^{12}$

Evaluation of stool-based methods is further complicated by the uncertain status of the IHA as a gold standard for past or present Schistosoma infections. Low specificity of the IHA in previous studies may in part be explained by use of a limited number of Kato-Katz smears or hatching of low sensitivity as the gold standard. Yu and others reported an IHA sensitivity and specificity of $80 \%$ and $48 \%$, respectively, using a comprehensive stool examination with duplicate Kato-Katz slides from 7 stool samples and hatching as the gold standard. ${ }^{11}$

The diagnostic strategy for schistosomiasis must change as control of the parasite progresses in an area. ${ }^{30}$ The two-step algorithm commonly used in China, with an antibody test followed by the Kato-Katz test of samples from patients who were seropositive, may not be appropriate as prevalence continues to decrease. In the present study, nearly half of the KatoKatz-positive cases would not have received treatment if this traditional algorithm was used. It remains uncertain whether the main problem in this situation is false-positive KatoKatz results or false-negative IHA results. More than twice as many persons would have received treatment if the PCR had replaced the Kato-Katz test in the diagnostic algorithm. Performance of the Kato-Katz test and to a lesser extent the hatching test is dependent upon the experience of the technician, while objective criteria can be made for a positive PCR. We have demonstrated the use of an inexpensive, non-commercial DNA extraction method applicable to local laboratories with limited resources. Amplification and detection can be centralized to larger facilities. As DNA-based diagnostic technologies are developed to become simpler and more affordable, they will be available in many laboratories, even in less affluent countries. However, it still is an expensive diagnostic alternative.

A satisfactory diagnostic algorithm for low-intensity S. japonicum infections in schistosomiasis-endemic areas has yet to be determined. More research in this field is needed to evaluate diagnostic algorithms with traditional tests and search for new methods. Diagnosing such infections will benefit patients and help to eliminate the parasite reservoir and assess if and to which degree transmission is still occurring. ${ }^{31}$ As prevalence decreases, additional measures to reduce transmission, such as infection control in the animal reservoir, improved sanitary conditions, and health education, will be of increasing importance. ${ }^{32}$

Received May 8, 2009. Accepted for publication June 10, 2009.
Acknowledgments: We thank Wu Weiduo, Chao Zhiguo, Xiao Xiang, Wang Qizhi, Zhang Gonghua, Liu Xiaoming, Chen Dalin, Pan Xinping, and the staff at Anhui Provincial Institute of Parasitic Diseases and Tongling Station of Schistosomiasis Control for collecting and processing samples.

Financial support: This study was supported by University Hospital of North Norway, Troms $\varnothing$, Norway.

Authors' addresses: Tore Lier, Gunnar S. Simonsen, Hanne H. Haukland, and Joachim Hegstad, Department of Microbiology and Infection Control, University Hospital of North Norway, PO Box 56, N-9038 Troms $\varnothing$, Norway, E-mails: tore.lier@unn.no, gunnar.skov .simonsen@unn.no, hanne.husom.haukland@unn.no, and joachim .hegstad@unn.no. Tianping Wang, Anhui Institute of Parasitic Diseases, 207 Dongjiao Road, Wuhu 241000, Anhui, Peoples Republic of China, E-mail: wangtianping@hotmail.com. Dabing Lu, Department of Infectious Diseases, Faculty of Medicine, Imperial College, London SW7 2AZ, United Kingdom, E-mail: dabing2001wyz@yahoo.com.cn. Birgitte J.Vennervald and Maria V. Johansen, DBL-Centre for Health Research and Development, Faculty of Life Sciences, Thorvaldsensvej 57, University of Copenhagen, DK-1871 Fredriksberg C, Denmark, E-mails: bjv@life.ku.dk and mvj@life.ku.dk.

\section{REFERENCES}

1. Chitsulo L, Engels D, Montresor A, Savioli L, 2000. The global status of schistosomiasis and its control. Acta Trop 77: 41-51.

2. Utzinger J, Zhou XN, Chen MG, Bergquist R, 2005. Conquering schistosomiasis in China: the long march. Acta Trop 96: 69-96.

3. Zhou XN, Guo JG, Wu XH, Jiang QW, Zheng J, Dang H, Wang XH, Xu J, Zhu HQ, Wu GL, Li YS, Xu XJ, Chen HG, Wang TP, Zhu YC, Qiu DC, Dong XQ, Zhao GM, Zhang SJ, Zhao NQ, Xia G, Wang LY, Zhang SQ, Lin DD, Chen MG, Hao Y, 2007. Epidemiology of schistosomiasis in the People's Republic of China, 2004. Emerg Infect Dis 13: 1470-1476.

4. Jiang Q, Wang L, Guo J, Chen M, Zhou X, Engels D, 2002. Morbidity control of schistosomiasis in China. Acta Trop 82: 115-125.

5. Engels D, Chitsulo L, Montresor A, Savioli L, 2002. The global epidemiological situation of schistosomiasis and new approaches to control and research. Acta Trop 82: 139-146.

6. Bergquist NR, 1992. Present aspects of immunodiagnosis of schistosomiasis. Mem Inst Oswaldo Cruz 87(Suppl 4): 29-38.

7. Katz N, Chaves A, Pellegrino J, 1972. A simple device for quantitative stool thick-smear technique in schistosomiasis mansoni. Rev Inst Med Trop Sao Paulo 14: 397-400.

8. Yu JM, de Vlas SJ, Yuan HC, Gryseels B, 1998. Variations in fecal Schistosoma japonicum egg counts. Am J Trop Med Hyg 59: 370-375.

9. Lin DD, Liu JX, Liu YM, Hu F, Zhang YY, Xu JM, Li JY, Ji MJ, Bergquist R, Wu GL, Wu HW, 2008. Routine Kato-Katz technique underestimates the prevalence of Schistosoma japonicum: a case study in an endemic area of the People's Republic of China. Parasitol Int 57: 281-286.

10. Wu G, 2002. A historical perspective on the immunodiagnosis of schistosomiasis in China. Acta Trop 82: 193-198.

11. Yu JM, de Vlas SJ, Jiang QW, Gryseels B, 2007. Comparison of the Kato-Katz technique, hatching test and indirect hemagglutination assay (IHA) for the diagnosis of Schistosoma japonicum infection in China. Parasitol Int 56: 45-49.

12. Zhou YB, Yang MX, Wang QZ, Zhao GM, Wei JG, Peng WX, Jiang QW, 2007. Field comparison of immunodiagnostic and parasitological techniques for the detection of schistosomiasis japonica in the People's Republic of China. Am J Trop Med Hyg 76: 1138-1143.

13. Pontes LA, Oliveira MC, Katz N, Dias-Neto E, Rabello A, 2003. Comparison of a polymerase chain reaction and the Kato-Katz technique for diagnosing infection with Schistosoma mansoni. Am J Trop Med Hyg 68: 652-656.

14. ten Hove RJ, Verweij JJ, Vereecken K, Polman K, Dieye L, van Lieshout L, 2008. Multiplex real-time PCR for the detection and quantification of Schistosoma mansoni and S. haematobium infection in stool samples collected in northern Senegal. Trans R Soc Trop Med Hyg 102: 179-185. 
15. Xia CM, Rong R, Lu ZX, Shi CJ, Xu J, Zhang HQ, Gong W, Luo W, 2009. Schistosoma japonicum: A PCR assay for the early detection and evaluation of treatment in a rabbit model. Exp Parasitol 121: $175-179$

16. Gobert GN, Chai M, Duke M, McManus DP, 2005. Copro-PCR based detection of Schistosoma eggs using mitochondrial DNA markers. Mol Cell Probes 19: 250-254.

17. Lier T, Simonsen GS, Haaheim H, Hjelmevoll SO, Vennervald BJ, Johansen MV, 2006. Novel real-time PCR for detection of Schistosoma japonicum in stool. Southeast Asian J Trop Med Public Health 37: 257-264.

18. Lier T, Johansen MV, Hjelmevoll SO, Vennervald BJ, Simonsen GS, 2008. Real-time PCR for detection of low intensity Schistosoma japonicum infections in a pig model. Acta Trop 105: 74-80.

19. Sørensen E, Drew AC, Brindley PJ, Bogh HO, Gasser RB, Qian BZ, Chiping Q, McManus DP, 1998. Variation in the sequence of a mitochondrial NADH dehydrogenase I gene fragment among six natural populations of Schistosoma japonicum from China. Int J Parasitol 28: 1931-1934.

20. Sørensen E, Bogh HO, Johansen MV, McManus DP, 1999. PCRbased identification of individuals of Schistosoma japonicum representing different subpopulations using a genetic marker in mitochondrial DNA. Int J Parasitol 29: 1121-1128.

21. Le TH, Humair PF, Blair D, Agatsuma T, Littlewood DT, McManus DP, 2001. Mitochondrial gene content, arrangement and composition compared in African and Asian schistosomes. Mol Biochem Parasitol 117: 61-71.

22. Altman DC, 1991. Practical Statistics for Medical Research. London: Chapman and Hall, 403-405.

23. Yogore MG, Lewert RM, Blas BL, 1983. Seroepidemiology of schistosomiasis japonica by ELISA in the Philippines. I. Underestimation by stool examination of the prevalence of infection in school children. Am J Trop Med Hyg 32: 1322-1334.

24. Zhou H, Ross AG, Hartel GF, Sleigh AC, Williams GM, McManus DP, Luo XS, He Y, Li YS, 1998. Diagnosis of schistosomiasis japonica in Chinese schoolchildren by administration of a questionnaire. Trans R Soc Trop Med Hyg 92: 245-250.

25. Hubbard A, Liang S, Maszle D, Qiu D, Gu X, Spear RC, 2002. Estimating the distribution of worm burden and egg excretion of Schistosoma japonicum by risk group in Sichuan Province, China. Parasitology 125: 221-231.

26. Wang T, Johansen MV, Zhang SQ, Wang FF, Wu WD, Zhang GH, Pan XP, Ju Y, Ørnbjerg N, 2005. Transmission of Schistosoma japonicum by humans and domestic animals in the Yangtze River valley, Anhui Province, China. Acta Trop 96: 198-204.

27. Ye XP, Fu YL, Wu ZX, Anderson RM, Agnew A, 1997. The effects of temperature, light and water upon the hatching of the ova of Schistosoma japonicum. Southeast Asian J Trop Med Public Health 28: 575-580.

28. Ye XP, Donnelly CA, Anderson RM, Fu YL, Agnew A, 1998. The distribution of Schistosoma japonicum eggs in faeces and the effect of stirring faecal specimens. Ann Trop Med Parasitol 92: 181-185.

29. Brinkmann UK, Powollik W, Werler C, Traore M, 1988. An evaluation of sampling methods within communities and the validity of parasitological examination techniques in the field. Trop Med Parasitol 39: 162-166.

30. Bergquist R, Johansen MV, Utzinger J, 2009. Diagnostic dilemmas in helminthology: what tools to use and when? Trends Parasitol 25: $151-156$.

31. World Health Organization, 2001. Report of the WHO Informal Consultation on Schistosomiasis in Low Transmission Areas: Control Strategies and Criteria for Elimination. April 10-13, 2000. Geneva: World Health Organization. WHO/CDS/CPE/ SIP/2001.1.

32. Wang LD, Chen HG, Guo JG, Zeng XJ, Hong XL, Xiong JJ, Wu XH, Wang XH, Wang LY, Xia G, Hao Y, Chin DP, Zhou XN, 2009. A strategy to control transmission of Schistosoma japonicum in China. N Engl J Med 360: 121-128. 